\title{
THE IONIZED CIRCUMSTELLAR ENVELOPES OF ORION SOURCE I AND THE BECKLIN-NEUGEBAUER OBJECT
}

\author{
R. L. Plambeck ${ }^{1}$, A. D. Bolatto ${ }^{2}$, J. M. Carpenter ${ }^{3}$, J. A. Eisner ${ }^{4}$, J. W. Lamb ${ }^{5}$, E. M. Leitch ${ }^{5,6}$, D. P. Marrone ${ }^{4}$, \\ S. J. Muchovej ${ }^{5}$, L. M. Pérez ${ }^{3}$, M. W. Pound ${ }^{2}$, P. J. Teuben ${ }^{2}$, N. H. Volgenau ${ }^{5}$, D. P. Woody ${ }^{5}$, \\ M. C. H. Wright ${ }^{1}$, AND B. A. ZAuderer ${ }^{7}$ \\ ${ }^{1}$ Radio Astronomy Lab, Hearst Field Annex, University of California, Berkeley, CA 94720, USA \\ 2 Department of Astronomy, University of Maryland, College Park, MD 20742, USA \\ ${ }^{3}$ Department of Astronomy, California Institute of Technology, Pasadena, CA 91125, USA \\ ${ }^{4}$ Department of Astronomy, University of Arizona, 933 North Cherry Avenue, Tucson, AZ 85721, USA \\ ${ }^{5}$ Owens Valley Radio Observatory, California Institute of Technology, P.O. Box 968, Big Pine, CA 93513, USA \\ ${ }^{6}$ Department of Astronomy and Astrophysics, University of Chicago, 5640 South Ellis Avenue, Chicago, IL 60637, USA \\ ${ }^{7}$ Department of Astronomy, Harvard University, Cambridge, MA 02138, USA \\ Received 2012 October 27; accepted 2013 January 15; published 2013 February 13
}

\begin{abstract}
The $229 \mathrm{GHz}(\lambda 1.3 \mathrm{~mm})$ radio emission from Orion-KL was mapped with up to 0 '. 14 angular resolution with CARMA, allowing measurements of the flux densities of Source I ("Srcl") and the Becklin-Neugebauer Object $(\mathrm{BN})$, the two most massive stars in this region. We find integrated flux densities of $310 \pm 45 \mathrm{mJy}$ for SrcI and $240 \pm 35 \mathrm{mJy}$ for BN. SrcI is optically thick even at $229 \mathrm{GHz}$. No trace of the $\mathrm{H} 30 \alpha$ recombination line is seen in its spectrum, although the $v_{2}=1,5(5,0)-6(4,3)$ transition of $\mathrm{H}_{2} \mathrm{O}, 3450 \mathrm{~K}$ above the ground state, is prominent. $\mathrm{SrcI}$ is elongated at position angle $140^{\circ}$, as in $43 \mathrm{GHz}$ images. These results are most easily reconciled with models in which the radio emission from SrcI arises via the $\mathrm{H}^{-}$free-free opacity in a $T<4500 \mathrm{~K}$ disk, as considered by Reid et al. By contrast, the radio spectrum of $\mathrm{BN}$ is consistent with $p^{+} / e^{-}$free-free emission from a dense $\left(n_{e} \sim 5 \times 10^{7} \mathrm{~cm}^{-3}\right)$, but otherwise conventional, hypercompact $\mathrm{H}$ II region. The source is becoming optically thin at $229 \mathrm{GHz}$, and the $\mathrm{H} 30 \alpha$ recombination line, at $V_{\mathrm{LSR}}=23.2 \pm 0.5 \mathrm{~km} \mathrm{~s}^{-1}$, is prominent in its spectrum. A Lyman continuum flux of $5 \times 10^{45}$ photons $\mathrm{s}^{-1}$, consistent with that expected from a $\mathrm{B}$ star, is required to maintain the ionization. Supplementary $90 \mathrm{GHz}$ observations were made to measure the $\mathrm{H} 41 \alpha$ and $\mathrm{H} 42 \alpha$ recombination lines toward BN. Published 43 and $86 \mathrm{GHz}$ data suggest that SrcI brightened with respect to BN over the 15 year period from 1994 to 2009.
\end{abstract}

Key words: ISM: individual objects (Orion-KL) - radio continuum: stars - radio lines: stars - stars: formation stars: individual (Becklin-Neugebauer-Object)

Online-only material: color figures

\section{INTRODUCTION}

The Kleinmann-Low Nebula in Orion is well-known as the nearest region of high mass star formation, $415 \mathrm{pc}$ away (Menten et al. 2007; Kim et al. 2008). It contains at least two massive young stars. One of these, the Becklin-Neugebauer Object (BN), has been studied extensively at infrared wavelengths (Scoville et al. 1983); it is thought to be a B star. The other object, Source I (hereafter, "Srcl"), is so heavily obscured by foreground dust that it is not directly visible in the infrared, although light reflected by the surrounding nebulosity provides a glimpse of its spectrum (Morino et al. 1998; Testi et al. 2010). SrcI is noteworthy because it is surrounded by a cluster of $\mathrm{SiO}$ masers, one of the few cases in which $\mathrm{SiO}$ masers are associated with a young star. It also is known to drive a bipolar outflow into the surrounding molecular cloud (Plambeck et al. 2009).

Remarkably, proper motion measurements show that SrcI and $\mathrm{BN}$ are recoiling from one another at $35-40 \mathrm{~km} \mathrm{~s}^{-1}$ (Rodríguez et al. 2005; Gómez et al. 2008; Goddi et al. 2011). Tracing the motions backward, Goddi et al. (2011) find that 560 years ago, the projected separation of these two stars in the plane of the sky was just $50 \pm 100 \mathrm{AU}$. An extensive system of "bullets," bow shocks, and "fingers," visible in lines of $\mathrm{H}_{2}, \mathrm{Fe}$ II, and $\mathrm{CO}$ (Allen \& Burton 1993; Zapata et al. 2011), also is centered on OrionKL. Proper motion measurements suggest that the fingers were created by an explosive event 500-1000 years ago (Doi et al.
2002; Bally et al. 2011). Thus, the currently favored paradigm for Orion-KL (Gómez et al. 2008; Bally et al. 2011; Goddi et al. 2011) postulates that $\mathrm{SrcI}$ and $\mathrm{BN}$ were ejected from a multiple system approximately 500 years ago, and that the ejection of the stars unbound the surrounding gas and circumstellar disks, creating the finger system.

BN's luminosity of $(0.8-2.1) \times 10^{4} L_{\odot}$ (De Buizer et al. 2012) suggests that it is a 10-15 $M_{\odot}$ star (Schaller et al. 1992). Estimates of the mass of SrcI are conflicting. SrcI is recoiling at about half the speed of $\mathrm{BN}$ in the rest frame of the Orion Nebula Cluster (Goddi et al. 2011); conservation of momentum then suggests that its mass is roughly $20 M_{\odot}$. On the other hand, if the $\mathrm{SiO}$ masers near $\mathrm{SrcI}$ are in Keplerian rotation about the star, then the inferred central mass is only $7 M_{\odot}$ (Matthews et al. 2010); this can be interpreted as a lower limit if the SiO-emitting gas is supported in part by radiation or magnetic pressure. SrcI could well be a compact binary with a semimajor axis of $<10 \mathrm{AU}$ (Gómez et al. 2008).

At $\mathrm{cm}$ and $\mathrm{mm}$ wavelengths, the continuum spectra of $\mathrm{BN}$ and SrcI are believed to be dominated by free-free emission from ionized circumstellar gas that is at least partially optically thick up to $100 \mathrm{GHz}$ (Plambeck et al. 1995). Observations at higher frequencies, where this emission should become optically thin, can be used to estimate the Lyman continuum fluxes from the stars, better constraining their masses. Such observations must be made with subarcsecond angular resolution in order to 
distinguish the compact circumstellar emission from bright but extended dust and molecular line emission from the surrounding molecular cloud.

Here, we describe high angular resolution $229 \mathrm{GHz}$ observations of Orion-KL with the Combined Array for Research in Millimeter-Wave Astronomy (CARMA) that cleanly resolve the emission from both stars. Surprisingly, we find that the emission from SrcI is optically thick even at $229 \mathrm{GHz}$, and we fail to find the $\mathrm{H} 30 \alpha$ recombination line in its spectrum. We argue that these results favor models in which the radio emission from SrcI originates from the $\mathrm{H}^{-}$opacity in a $T<4500 \mathrm{~K}$ disk.

\section{OBSERVATIONS}

The spectra of SrcI and BN presented here were obtained from a single night's observation of Orion made with the CARMA B-array on 2011 January 6. Fourteen antennas were used; projected antenna separations ranged from 50 to $700 \mathrm{k} \lambda$. The phase center was $05^{\mathrm{h}} 35^{\mathrm{m}} 14.505,-05^{\circ} 22^{\prime} 30^{\prime}$. 45 (J2000). The weather was excellent - the atmospheric opacity at $225 \mathrm{GHz}$ was 0.10 , and the rms atmospheric phase fluctuations were $65 \mu \mathrm{m}$ on a $100 \mathrm{~m}$ baseline. Double sideband system temperatures for the dual-polarization receivers were 100-200 K, scaled to outside the atmosphere. For each polarization, the correlator was configured to observe 3 overlapping $500 \mathrm{MHz}$ windows (covering the frequency range $225.8-227.2 \mathrm{GHz}$ in the receivers' lower sideband and $231.4-232.8 \mathrm{GHz}$ in the upper sideband, with $12.5 \mathrm{MHz}$ wide channels), plus a single $250 \mathrm{MHz}$ window (covering 226.65-226.90 GHz in the lower sideband and 231.75-232.00 GHz in the upper sideband, with $3.125 \mathrm{MHz}$ resolution). Upper and lower sideband signals are separated in the correlator by phase-switching.

The antenna gains were derived from observations of the calibrators 0423-013 (2.4 Jy) and 0607-085 (1.05 Jy) that were interleaved with the Orion scans every 10 minutes. To minimize decorrelation from atmospheric phase fluctuations, a self-calibration interval of $30 \mathrm{~s}$ was used. The calibrator flux densities were established from observations of the primary flux standard, Uranus. Because Uranus is heavily resolved on these baselines, only antenna pairs with separations $<200 \mathrm{k} \lambda$ were used for flux calibration. Antenna gain solutions were applied to the Orion data after smoothing to a 10 min interval (scalar averaging the amplitude corrections). Only data with antenna separations greater than $250 \mathrm{k} \lambda$ were used for the Orion maps, yielding a $0.28 \times 0.21$ synthesized beam at P.A. $-27^{\circ}$.

With $0^{\prime} .25$ angular resolution, almost all of the spectral line emission from the Orion Hot Core and surrounding molecular cloud is resolved out. Nevertheless, to ensure that continuum flux densities were not contaminated by residual line emission or absorption, we made maps of all of the spectral channels, and flagged those (28 out of 234) with anomalously high $(>2 \times$ average) rms noise; the noisy channels coincided with strong spectral lines. We generated a primary-beam corrected continuum map from the remaining channels, using multifrequency synthesis to avoid radial smearing.

To help correct the Orion data for atmospheric decorrelation, a single phase-only self-calibration was performed on the source itself. This means that after cleaning the continuum map in the normal way, the brightest pixels in the clean component list were used as a source model to refine the antenna-based phases. A time interval of two minutes was used, short enough to track the most significant atmospheric phase variations, but long enough to obtain a signal-to-noise ratio of $\geqslant 1$ on each of the 91 available baselines, allowing a robust least squares fit for the 13 antenna- based phases. Applying the self-calibrated phase corrections increased the source flux densities by about $50 \%$. The measured rms noise in the final 0 .'25 resolution map is $4 \mathrm{mJy}_{\text {beam }}{ }^{-1}$.

Even higher resolution images of Orion were obtained in 2009 February using the CARMA A-array, with antenna separations of up to $1.7 \mathrm{~km}(1350 \mathrm{k} \lambda)$. These observations utilized older, single-polarization receivers and a $1.5 \mathrm{GHz}$ bandwidth correlator. For these data, the CARMA Paired Antenna Calibration System ("C-PACS"; Pérez et al. 2010) was used as an adaptive optics scheme to correct for blurring by atmospheric phase fluctuations. C-PACS placed the 8 CARMA 3.5 meter telescopes next to a subset of the $10 \mathrm{~m}$ and $6 \mathrm{~m}$ telescopes, including those at the extremes of the array. As the $6 \mathrm{~m}$ and $10 \mathrm{~m}$ telescopes observed Orion at $229 \mathrm{GHz}$, the $3.5 \mathrm{~m}$ antennas observed the nearby (1.6 away) quasar $0541-056$ at $31 \mathrm{GHz}$ in order to monitor the atmospheric phase variations above each station. The atmospheric phase delay to each $3.5 \mathrm{~m}$ telescope was derived by self-calibration on 0541-056 every $12 \mathrm{~s}$. These antenna-based phases were scaled up by the ratio of the observing frequencies $(229 / 31=7.4)$ and applied to the mm data. The C-PACS calibration accuracy is limited by the angular separation of the $\mathrm{cm}$ calibrator and the $\mathrm{mm}$ source, which causes the $\mathrm{cm}$ and $\mathrm{mm}$ beams to probe different paths through the atmosphere; by the signal to noise ratio on the $31 \mathrm{GHz}$ calibrator; and by the airmass.

A continuum map was generated from the A-array data using only baselines longer than $250 \mathrm{k} \lambda$. The synthesized beam was $0^{\prime \prime} .15 \times 0^{\prime \prime} .13$ at P.A. $14^{\circ}$. Applying the C-PACS corrections more than doubled the signal-to-noise ratio of this map. As with the B-array map, we then performed a single phase-only self-calibration, with a two minute interval, to help correct for residual atmospheric phase fluctuations; this doubled the flux densities of the compact sources. The measured rms noise in the final 0.14 resolution map is $2.6 \mathrm{mJy}^{\text {beam }}{ }^{-1}$.

The integrated flux densities measured from the 0 '. 14 and 0.25 resolution maps were 240 and $255 \mathrm{mJy}$ for BN, 310 and $370 \mathrm{mJy}$ for SrcI. Although the discrepancy in the two SrcI flux densities could conceivably be caused by time variability of the source (see Section 4.2.3), it is more likely attributable to differences in the sampling of visibilities by the A and B arrays, which lead to different sidelobe structure from the adjacent Orion Hot Core. We will use the A-array flux densities for the remainder of this paper because the higher resolution data should more effectively filter out emission from the Hot Core. We estimate that the absolute flux scale is accurate within $\pm 15 \%$.

Finally, supplementary $3 \mathrm{~mm}$ observations were made with the A-array in 2009 February to obtain spectra of the H41 $\alpha$ $(92.034 \mathrm{GHz})$ and $\mathrm{H} 42 \alpha(85.688 \mathrm{GHz})$ recombination lines toward $\mathrm{BN}$. Both of the recombination lines and the strong $\mathrm{SiO}$ maser at $86.243 \mathrm{GHz}$ were measured simultaneously. The data were self-calibrated on the maser using a $10 \mathrm{~s}$ interval. The spectra of the two recombination lines were averaged together to improve the signal-to-noise ratio.

\section{RESULTS}

The left hand panel in Figure 1 shows the $00^{\prime} .25$ resolution continuum map from the B-array observations, overlaid on a 0.83 resolution image obtained with the CARMA C-array (Eisner et al. 2008). SrcI and BN are clearly distinguishable only in the higher resolution map; extended emission from dust and molecular lines dominates the lower resolution image.

The upper sideband spectra of BN and SrcI are shown in the right hand panels of Figure 1. The $\mathrm{H} 30 \alpha$ recombination line at $231.901 \mathrm{GHz}$ is prominent in the spectrum of $\mathrm{BN}$, but is not 

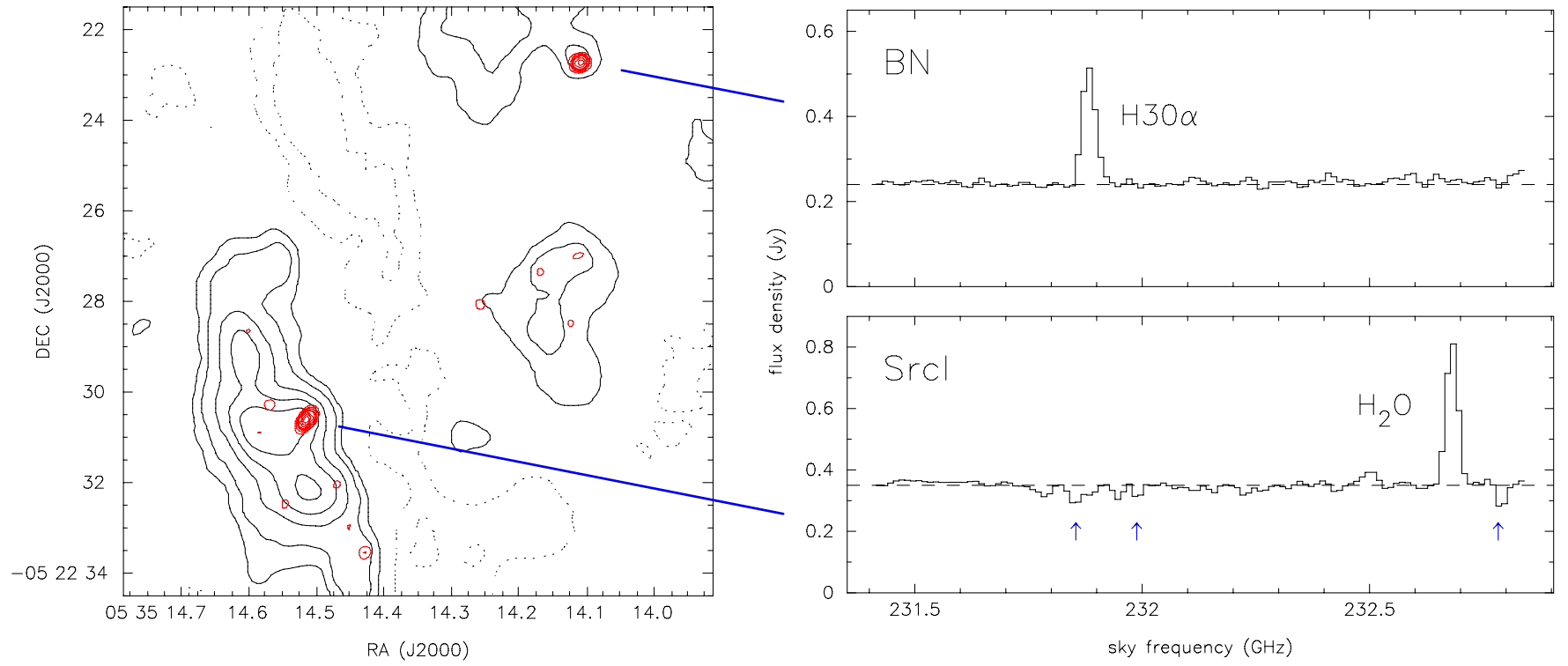

Figure 1. Left: $229 \mathrm{GHz}$ continuum maps of Orion-KL from CARMA C- and B-array data. Black contours show the 0"' 83 resolution C-array map; the contour levels are $-100,-50,50,100,200,300$, and 400 mJy beam $^{-1}$. Negative contours are attributable to poorly sampled extended emission. Red contours show the 0 '. 25 resolution B-array image; contour levels are 20,40,60, 80, 100, 150, 200, and $250 \mathrm{mJy}^{\text {beam }}{ }^{-1}$. Extended emission is almost entirely filtered out in this map; the most negative value is $-30 \mathrm{mJy}_{\text {beam }}{ }^{-1}$. Right: spectra of BN and SrcI near $232 \mathrm{GHz}$ obtained from the $0^{\prime \prime} 25$ resolution image. Dashed lines indicate the continuum levels. The spectra have been Hanning smoothed to $30 \mathrm{~km} \mathrm{~s}^{-1}$ velocity resolution. The $\mathrm{H} 30 \alpha$ line is detected toward $\mathrm{BN}$, the $\mathrm{H}_{2} \mathrm{O} v_{2}=1,5(5,0)-6(4,3)$ line toward SrcI. Arrows in the SrcI spectrum indicate weak absorption features from transitions of ethyl cyanide, dimethyl ether, and methanol.
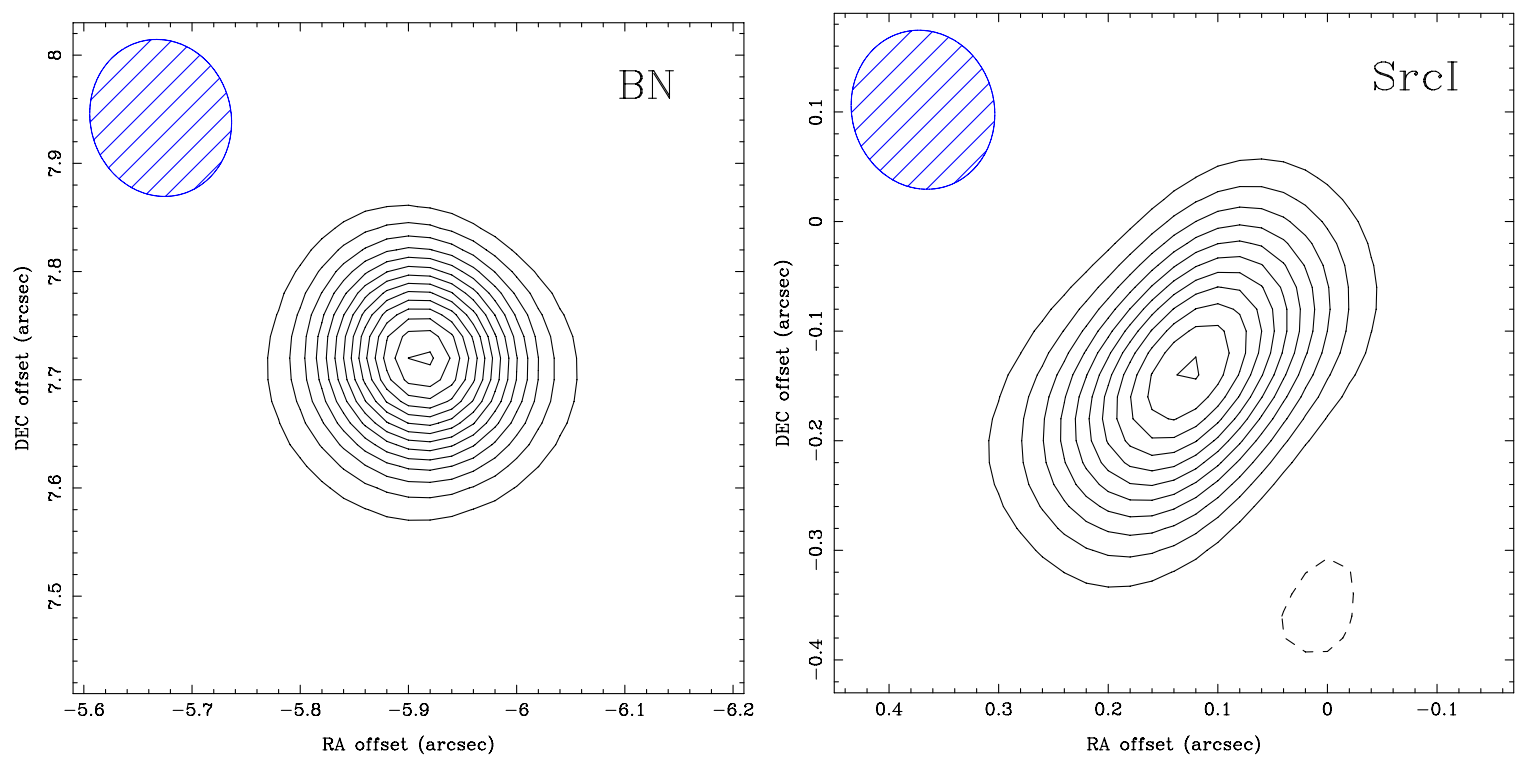

Figure 2. Images of BN and SrcI at $229 \mathrm{GHz}$ from the CARMA A-array data. Each box is $00^{\prime \prime} 6$ on a side. The lowest contour and the contour interval is $15 \mathrm{mJy}$ beam ${ }^{-1}$. The rms noise level is $2.6 \mathrm{mJy}$ beam ${ }^{-1}$. The $0^{\prime} \cdot 15 \times 00^{\prime \prime} 13$ synthesized beam is shown by a hatched ellipse in the upper left corner of each image. SrcI is extended at P.A. $140^{\circ}$, as in $43 \mathrm{GHz}$ VLA images (Reid et al. 2007; Goddi et al. 2011).

detected toward SrcI. The bright line at $232.687 \mathrm{GHz}$ in SrcI's spectrum is the $\mathrm{v}_{2}=1,5(5,0)-6(4,3)$ transition of $\mathrm{H}_{2} \mathrm{O}$. Weak absorption features toward SrcI are due to transitions of ethyl cyanide, dimethyl ether, and methanol. No emission lines were detected toward either source in the lower sideband.

Figure 2 displays the 0'.14 resolution images of BN and SrcI from the A-array. BN is nearly circularly symmetric, while SrcI is elongated. Table 1 summarizes the results of Gaussian fits to the positions, flux densities, and deconvolved sizes. The $229 \mathrm{GHz}$ source positions match the $43 \mathrm{GHz}$ positions measured in 2009 January by Goddi et al. (2011) within 0'015. The deconvolved size of $\mathrm{SrcI}$ at $229 \mathrm{GHz}, 0^{\prime} \cdot 20 \times 00^{\prime} 03$ at P.A. $140^{\circ}$, is similar to the sizes measured at $8.4 \mathrm{GHz}\left(0^{\prime \prime} 19 \times<00^{\prime \prime} 15\right.$ at P.A. $136^{\circ}$; Gómez et al. 2008) and at $43 \mathrm{GHz}\left(0^{\prime \prime} 23 \times 0^{\prime \prime} \cdot 12\right.$ at P.A. $142^{\circ}$; Goddi et al. 2011), although the source appears to be thinner along its minor axis at $229 \mathrm{GHz}$.

The flux density we measure for $\mathrm{BN}$ in the 0 ' 14 resolution map is roughly $2 \times$ higher than the value recently reported by Galván-Madrid et al. (2012) from an analysis of ALMA Band 6 Science Verification data. These early ALMA data had a $1^{\prime \prime} .3 \times 00^{\prime} 6$ synthesized beam. We suspect that the flux density of BN is depressed in the ALMA map because of negative sidelobes from poorly sampled extended emission. The same problem affects the 0'.83 resolution CARMA image shown in 
Table 1

Source Parameters for SrcI and BN at $229 \mathrm{GHz}$

\begin{tabular}{lccccc}
\hline \hline Source & $\begin{array}{c}\text { R.A. } \\
(\mathrm{h} \mathrm{m} \mathrm{s})\end{array}$ & $\begin{array}{c}\text { Decl. } \\
\left({ }^{\prime \prime}{ }^{\prime \prime}\right)\end{array}$ & $\begin{array}{c}\text { Peak Flux Density } \\
(\mathrm{mJy})\end{array}$ & $\begin{array}{c}\text { Integrated Flux Density } \\
(\mathrm{mJy})\end{array}$ & Deconvolved Size \\
\hline SrcI & 053514.514 & -052230.59 & $170 \pm 25$ & $310 \pm 45$ & $0.20 \times 0^{\prime \prime} .03$ at P.A. $140^{\circ}$ \\
BN & 053514.109 & -052222.73 & $215 \pm 30$ & $240 \pm 35$ & $0.06 \times 0^{\prime \prime} 04$ at P.A. $90^{\circ}$ \\
\hline
\end{tabular}

Notes. All parameters were measured from the 0.' 14 resolution map. Positions are for epoch 2009.1.

Table 2

Integrated Flux Densities for SrcI and BN

\begin{tabular}{lcccr}
\hline \hline $\begin{array}{l}\text { Frequency } \\
(\mathrm{GHz})\end{array}$ & $\begin{array}{c}S(\mathrm{SrcI}) \\
(\mathrm{mJy})\end{array}$ & $\begin{array}{c}S(\mathrm{BN}) \\
(\mathrm{mJy})\end{array}$ & Epoch & References \\
\hline 4.8 & $\ldots$ & $2.2 \pm 0.8$ & 1990 & 1 \\
8.4 & $1.1 \pm 0.2$ & $4.5 \pm 0.7$ & 1994.3 & 2 \\
8.4 & $1.2 \pm 0.1$ & $4.8 \pm 0.1$ & 2006.4 & 3 \\
15 & $\ldots$ & $9.1 \pm 0.6$ & 1981.6 & 4 \\
15 & $\ldots$ & $9.4 \pm 1.0$ & 1983.7 & 4 \\
15 & $1.54 \pm 0.18$ & $6.19 \pm 0.19$ & 1986.3 & 5 \\
15 & $1.6 \pm 0.4$ & $6.8 \pm 1.5$ & 1990 & 1 \\
22 & $\ldots$ & $13.6 \pm 1.1$ & 1983.7 & 4 \\
22 & $5.7 \pm 0.9$ & $16.57 \pm 0.7$ & 1991.5 & 6 \\
43 & $13 \pm 2$ & $31 \pm 4.7$ & 1994.3 & 2 \\
43 & $10.8 \pm 0.6$ & $28.0 \pm 0.6$ & 1994.9 & 7 \\
43 & 13 & $26.4 \pm 0.7$ & 2000.9 & 3,8 \\
43 & $14.5 \pm 0.7$ & $28.6 \pm 0.6$ & 2007.9 & 9 \\
43 & $11 \pm 2$ & $23 \pm 2$ & 2009.0 & 10 \\
86 & $34 \pm 5$ & $84 \pm 10$ & 1995.0 & 11 \\
89 & $50 \pm 5$ & $91 \pm 9$ & 2009.0 & 12 \\
229 & $310 \pm 45$ & $240 \pm 35$ & 2009.1 & 13 \\
348 & $320 \pm 48$ & $\ldots$ & 2004.1 & 14 \\
690 & $6700 \pm 3200$ & $\ldots$ & 2005.1 & 15 \\
\hline & & & & \\
\hline
\end{tabular}

References. (1) Felli et al. 1993b; (2) Menten \& Reid 1995; (3) Gómez et al. 2008; (4) Garay et al. 1987; (5) Felli et al. 1993a; (6) Forbrich et al. 2008; (7) Chandler \& Wood 1997; (8) Reid et al. 2007; (9) Rodríguez et al. 2009; (10) Goddi et al. 2011; (11) Plambeck et al. 1995; (12) Friedel \& Widicus Weaver 2011 (assuming 10\% absolute calibration accuracy); (13) this paper; (14) Beuther et al. 2004; (15) Beuther et al. 2006.

Figure 1; the peak intensity at the position of $\mathrm{BN}$ in this low resolution image is $\sim 125 \mathrm{mJy}_{\text {beam }}^{-1}$. The higher resolution CARMA maps are free of deep negative sidelobes, allowing more reliable flux density measurements of compact sources.

\section{DISCUSSION}

Figure 3 shows the radio continuum spectra of $\mathrm{BN}$ and $\mathrm{SrcI}$ from $\mathrm{cm}$ to submillimeter wavelengths. Numerical values are given in Table 2. For $\mathrm{BN}$, we also plot the peak intensities of the $\mathrm{H} 30 \alpha$ and $\mathrm{H} 41 / 42 \alpha$ recombination lines measured with CARMA, and the H53 $\alpha$ line measured with the Very Large Array (VLA; Rodríguez et al. 2009).

\subsection{BN}

The continuum spectrum of $\mathrm{BN}$ is typical of free-free emission from a hypercompact $\mathrm{H}$ II region. The flux density scales as $v^{1.3}$, indicating that optically thick emission has a larger angular extent at lower frequencies, either because the ionized gas is clumpy (Ignace \& Churchwell 2004) or because the electron density declines smoothly with radius (Wright \& Barlow 1975). The spectrum begins to flatten at $229 \mathrm{GHz}$, indicating that the source is becoming optically thin at higher frequencies.

\subsubsection{Model Spectra}

To model the spectrum, we assume that the emission region is spherically symmetric, with a uniform electron temperature $T_{e}$ and an electron density that is constant, $n_{e}(r)=n_{0}$, inside the core radius $r_{c}$, then declines as $n_{e}(r)=n_{0}\left(r / r_{c}\right)^{\alpha}$ at larger radii. For each projected radius $x$, we numerically integrate along the line of sight to compute the emission measure $\operatorname{EM}(x)=\int n_{e}^{2} d z \mathrm{pc} \mathrm{cm}^{-6}$. The brightness temperatures at this projected radius in the continuum and at the peak of a recombination line are then $T_{C}(x)=T_{e}\left[1-\exp \left(-\tau_{C}\right)\right]$ and $T_{L}(x)=T_{e}\left[1-\exp \left(-\tau_{C}-\tau_{L}\right)\right]$, where the continuum and line opacities are approximated as (Rohlfs \& Wilson 2000)

$$
\begin{aligned}
& \tau_{C}(x)=8.23 \times 10^{-2} T_{e}^{-1.35} v_{\mathrm{GHz}}^{-2.1} \operatorname{EM}(x) \\
& \tau_{L}(x)=1.92 \times 10^{3} T_{e}^{-2.5} \operatorname{EM}(x) \Delta v_{\mathrm{kHz}}^{-1} .
\end{aligned}
$$

We integrate the brightness temperatures over circular annuli to obtain flux densities, assuming a distance to the source of 415 pc.

The model BN spectra shown by smooth curves in Figure 3 assumed $T_{e}=8000 \mathrm{~K}, n_{0}=5 \times 10^{7} \mathrm{~cm}^{-3}, r_{c}=7.4 \mathrm{AU}$, $\alpha=-3.5$, and recombination line velocity widths of $30 \mathrm{~km} \mathrm{~s}^{-1}$ for all transitions. The model does not take into account systematic velocity gradients or pressure broadening of the recombination lines. The model parameters were determined by trial and error, and are not a formal fit to the data. To first order, however, the turnover frequency sets $n_{0}$, the spectral slope sets $\alpha$, and the absolute flux densities set $r_{c}$.

\subsubsection{Excitation Parameter}

The model parameters above yield an excitation parameter $U=r_{c}\left(n_{e} n_{H}\right)^{1 / 3}=5 \mathrm{pc} \mathrm{cm}^{-2}$, which serves as a measure of the flux of ionizing photons from the central star. This corresponds to the excitation parameter expected for a main sequence $\mathrm{B}$ star with Lyman continuum flux $5 \times 10^{45}$ photons $\mathrm{s}^{-1}$ and total luminosity $(0.5-1) \times 10^{4} L_{\odot}($ Panagia 1973). The ionizing flux is comparable to the value derived by Scoville et al. (1983) from infrared data, and the luminosity is comparable with the bolometric luminosity obtained by De Buizer et al. (2012) from 6-37 $\mu \mathrm{m}$ SOFIA observations.

\subsubsection{Recombination Lines}

Figure 4 displays the recombination line profiles toward BN. $\mathrm{H} 30 \alpha$ and $\mathrm{H} 41 / 42 \alpha$ (the average of the $\mathrm{H} 41 \alpha$ and $\mathrm{H} 42 \alpha$ lines) were observed with CARMA, and H53 $\alpha$ with the VLA (Rodríguez et al. 2009). The $\mathrm{H} 30 \alpha$ spectrum was measured using the $250 \mathrm{MHz}$ wide correlator window with $4 \mathrm{~km} \mathrm{~s}^{-1}$ channel spacing; it has been Hanning smoothed to $8 \mathrm{~km} \mathrm{~s}^{-1}$ resolution. Line parameters and uncertainties derived from Gaussian fits to the spectra are summarized in Table 3. The $\mathrm{H} 53 \alpha$ line is at a slightly lower velocity and is noticeably wider than the higher frequency lines. 

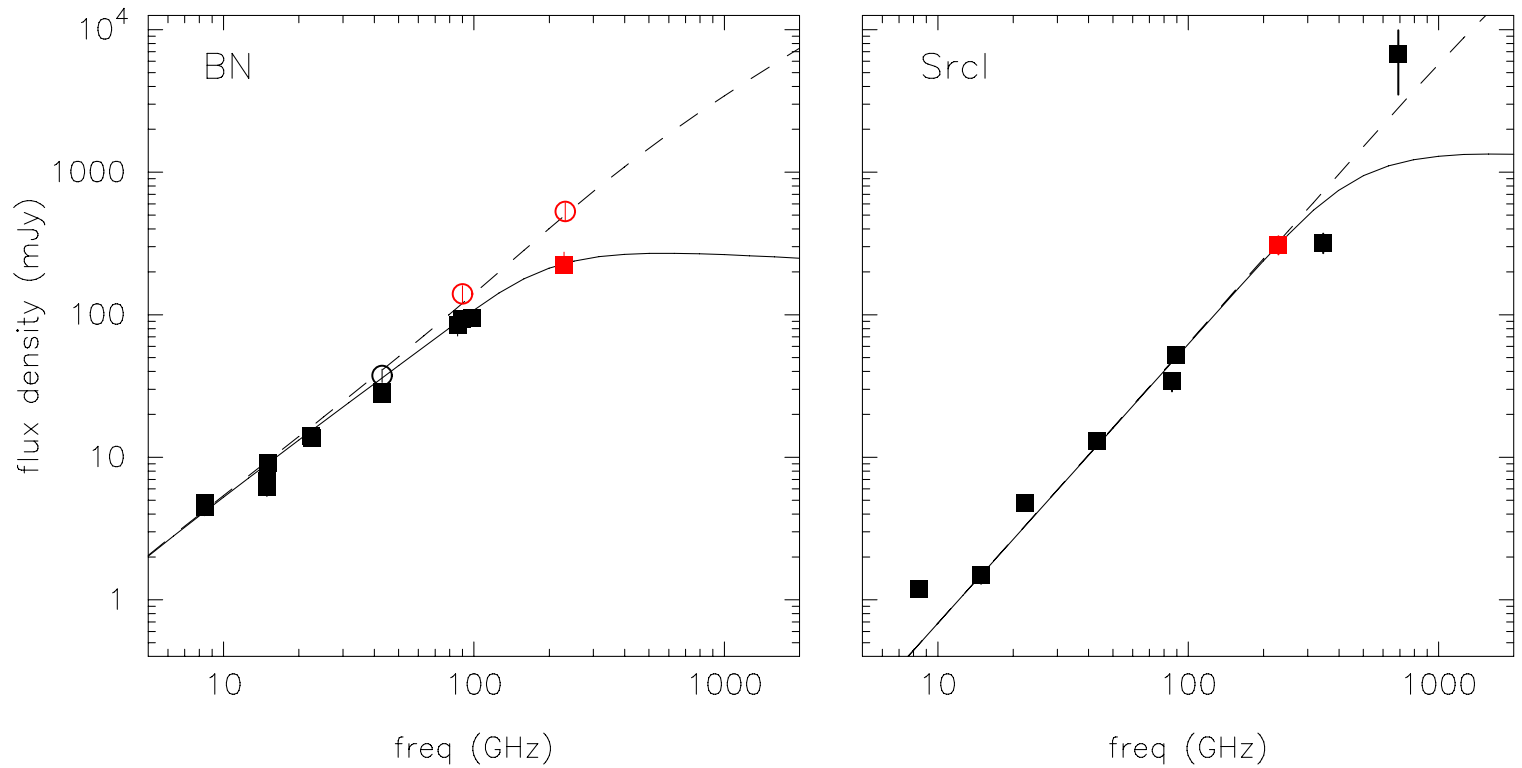

Figure 3. Radio spectra of BN and SrcI. Squares indicate continuum flux densities; circles, flux densities at the peak of recombination lines. CARMA results presented in this paper are shown as red in the online edition. Solid and dashed lines show, respectively, the continuum and recombination line flux densities predicted by the models described in Sections 4.1.1 and 4.2.1.

(A color version of this figure is available in the online journal.)

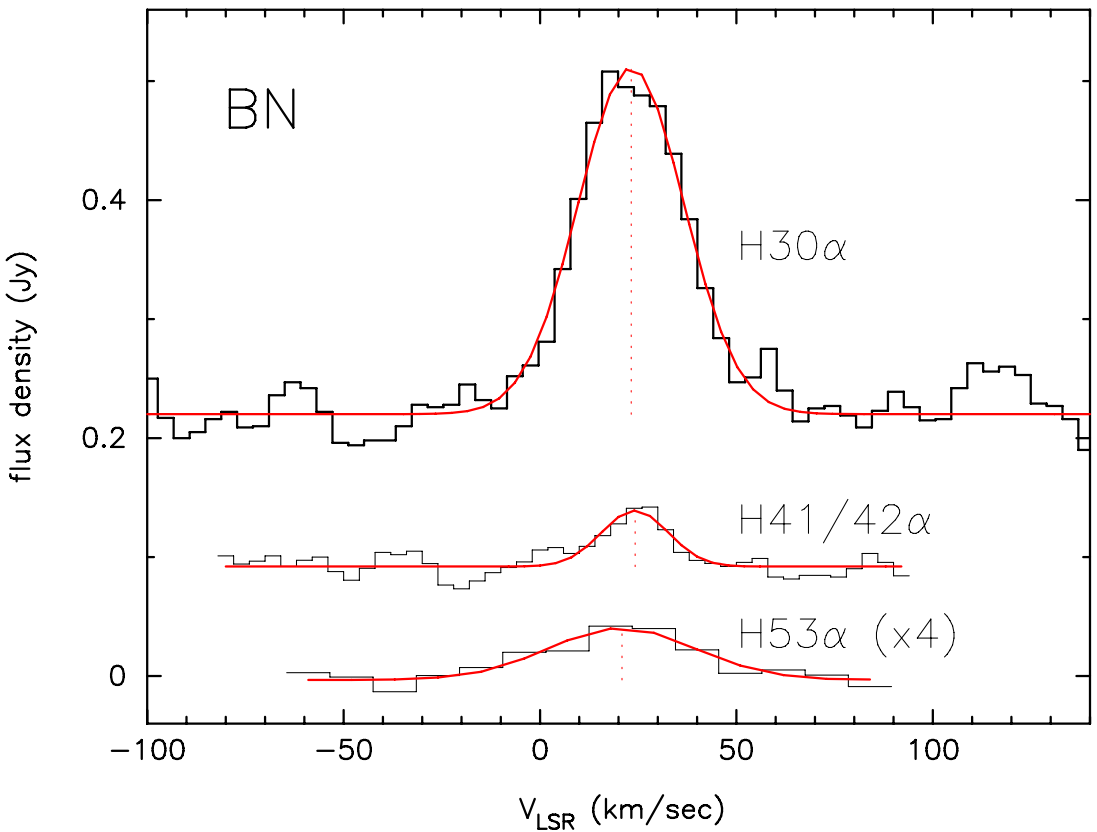

Figure 4. Hydrogen recombination lines observed toward BN. $\mathrm{H} 30 \alpha$ and $\mathrm{H} 41 / 42 \alpha$ (the average of the two spectra) were observed with CARMA; H53 $\alpha$, with the VLA (Rodríguez et al. 2009). The H53 $\alpha$ flux densities are scaled up by 4 for better visibility. Smooth curves show Gaussian fits to the spectra.

(A color version of this figure is available in the online journal.)

For the electron densities $n_{e}>10^{7} \mathrm{~cm}^{-3}$ that we infer for BN, collisional broadening of recombination lines can be significant. Collisional broadening is most important for high- $n$ transitions because the diameter of a hydrogen atom is proportional to $n^{2}$-an atom in the $n=53$ level is $0.3 \mu \mathrm{m}$ across! The collisionbroadened linewidths are given by the approximate formula (Brocklehurst \& Seaton 1972)

$$
\Delta v_{c} / 2=4.7 c / v(n / 100)^{4.4}\left(10^{4} / T_{e}\right)^{0.1} n_{e} .
$$

For $n_{e}=5 \times 10^{7} \mathrm{~cm}^{-3}$ and $T_{e}=8000 \mathrm{~K}, \Delta v_{c} \sim 200 \mathrm{~km} \mathrm{~s}^{-1}$ for $\mathrm{H} 53 \alpha, 30 \mathrm{~km} \mathrm{~s}^{-1}$ for $\mathrm{H} 41 \alpha$, and $3 \mathrm{~km} \mathrm{~s}^{-1}$ for $\mathrm{H} 30 \alpha$. These widths must be convolved with the expected $\sim 20 \mathrm{~km} \mathrm{~s}^{-1}$ wide thermal profiles, plus any additional Doppler broadening from turbulent motions.

Since the observed width of the H53 $\alpha$ line is much less than $200 \mathrm{~km} \mathrm{~s}^{-1}$, most of the $\mathrm{H} 53 \alpha$ emission that is detected must originate more than $\sim 12 \mathrm{AU}$ from the star, in the lower density halo where $n_{e}<10^{7} \mathrm{~cm}^{-3}$. The line is slightly blueshifted relative to the other transitions, as expected if this gas is expanding. The $\mathrm{H} 30 \alpha$ line is little affected by pressure broadening or by continuum opacity, so its central velocity, $23.2 \mathrm{~km} \mathrm{~s}^{-1}$, is the best indicator of the $V_{\mathrm{LSR}}$ of BN. We attribute the anomalously narrow width observed for the 
Table 3

Recombination Line Parameters for BN

\begin{tabular}{lcccc}
\hline \hline Line & $\begin{array}{c}\text { Freq } \\
(\mathrm{GHz})\end{array}$ & $\begin{array}{c}\mathrm{S}_{\text {peak }}{ }^{\mathrm{a}} \\
(\mathrm{mJy})\end{array}$ & $\begin{array}{c}V_{\text {LSR }} \\
\left(\mathrm{km} \mathrm{s}^{-1}\right)\end{array}$ & $\begin{array}{c}\text { FWHM } \\
\left(\mathrm{km} \mathrm{s}^{-1}\right)\end{array}$ \\
\hline $\mathrm{H} 53 \alpha^{\mathrm{b}}$ & 42.952 & $10.4 \pm 1.1$ & $20.1 \pm 2.1$ & $39.0 \pm 4.9$ \\
$\mathrm{H} 42 \alpha$ & 85.688 & $47 \pm 5^{\mathrm{c}}$ & $24.2 \pm 1.1^{\mathrm{c}}$ & $20.0 \pm 2.7^{\mathrm{c}}$ \\
$\mathrm{H} 41 \alpha$ & 92.034 & $291 \pm 10$ & $23.2 \pm 0.5$ & $31.8 \pm 1.3$ \\
$\mathrm{H} 30 \alpha$ & 231.901 & & & \\
\hline
\end{tabular}

Notes.

a Peak flux density of the line above the continuum level.

b Rodríguez et al. (2009).

${ }^{c}$ Fit to average of the $\mathrm{H} 41 \alpha$ and $\mathrm{H} 42 \alpha$ line profiles.

$\mathrm{H} 41 / 42 \alpha$ transition to poor signal to noise; more sensitive spectra of all of these recombination lines would be useful in further constraining models of the electron density in BN.

We do not detect the $231.995 \mathrm{GHz}$ helium recombination line toward BN. The absence of this line is consistent with the identification of BN as a B-star. In the $30 \mathrm{~km} \mathrm{~s}^{-1}$ resolution BN spectrum in Figure 1, the $2 \sigma$ upper limit on the intensity of the $\mathrm{He} 30 \alpha$ line is $\sim 12 \mathrm{mJy}$, while the intensity of the $\mathrm{H} 30 \alpha$ line is $270 \mathrm{mJy}$; thus, $\tau(\mathrm{He} 30 \alpha) / \tau(\mathrm{H} 30 \alpha)<0.045$. If helium were ionized over the full volume of the $\mathrm{H}$ II region, then the opacity ratio would be comparable to the $\mathrm{He} / \mathrm{H}$ abundance ratio, $\sim 0.1$. Thus, we can say that the $\mathrm{He}^{+}$zone, if it exists, fills less than half of the volume of the $\mathrm{H}$ II region. This places an upper limit of roughly 0.05 on the ratio of helium-ionizing to hydrogenionizing photons from the star (Mezger et al. 1974, Figure 2), consistent with the ratio expected for B-stars, but not for O9 or more massive stars (Mezger et al. 1974; Vacca et al. 1996).

\section{2. $\mathrm{SrcI}$}

According to the data in Figure 3, the flux density of SrcI scales approximately as $v^{2}$ from 43 to $229 \mathrm{GHz}$, suggesting that over this frequency range the source is an optically thick black body with constant angular size. Even if the absolute calibrations are wrong, the absence of a detectable $\mathrm{H} 30 \alpha$ line rules out the possibility that the emission is optically thin $p^{+} / e^{-}$bremsstrahlung, unless the $\mathrm{H} 30 \alpha$ line is unexpectedly broad $\left(>300 \mathrm{~km} \mathrm{~s}^{-1}\right)$. For a linewidth of $30 \mathrm{~km} \mathrm{~s}^{-1}$, comparable to the velocity span of the $\mathrm{SrcI} \mathrm{SiO}$ masers, the line to continuum opacity ratio $\tau_{L} / \tau_{C} \sim 3$ from Equations (1) and (2), and the line should easily have been detected if $\tau_{C}<2$.

Our data cannot easily be reconciled with the $348 \mathrm{GHz}$ flux density of $320 \pm 48$ mJy measured with the Submillimeter Array (SMA) (Beuther et al. 2004), which falls below plausible extrapolations from the lower frequency points. Such a low $348 \mathrm{GHz}$ flux density would imply that the free-free emission from $\mathrm{SrcI}$ is somewhat optically thin at $229 \mathrm{GHz}$, in which case we should have detected the $\mathrm{H} 30 \alpha$ line. Possibly the $348 \mathrm{GHz}$ SrcI measurement was corrupted by negative sidelobes from the bright Hot Core clumps 1-2" east of SrcI, or possibly SrcI is time-variable (see Section 4.2.3). The $690 \mathrm{GHz}$ flux density measured with the SMA, 6.7 \pm 3.2 Jy (Beuther et al. 2006), lies above the $v^{2}$ extrapolation from lower frequencies, but this is easily explained if there is thermal emission from dust within the synthesized beam.

\subsubsection{An Optically Thick Hypercompact H II Region?}

If $\mathrm{SrcI}$ is a conventional hypercompact $\mathrm{H}$ II region, then it must have an extraordinarily high emission measure, $>3 \times$
$10^{11} \mathrm{pc} \mathrm{cm}^{-6}$, in order to remain optically thick at $229 \mathrm{GHz}$. We are able reproduce the observed continuum flux densities with the simple spherically symmetric model described in Section 4.1.1 if we assume that the ionized region has radius $r_{c}=$ 7.5 AU, electron temperature $T_{e}=8000 \mathrm{~K}$, and uniform electron density $n_{e}=1.5 \times 10^{8} \mathrm{~cm}^{-3}$. The resulting continuum spectrum is shown by the solid curve in Figure 3; the dashed curve shows the recombination line intensities predicted by the model. The H II region is constrained to have a sharp outer edge-any halo of lower density plasma would lead to excess emission at $\mathrm{cm}$ wavelengths. Collisional broadening of the $\mathrm{H} 30 \alpha$ line is predicted to be of the order of $10 \mathrm{~km} \mathrm{~s}^{-1}$, not enough to smear out the line. The excitation parameter $U \sim 10 \mathrm{pc} \mathrm{cm}^{-2}$ computed from the radius and electron density corresponds to an ionizing flux of $4 \times 10^{46}$ photons $\mathrm{s}^{-1}$, consistent with a B0 to B1 main sequence star with luminosity $\sim 10^{4} L_{\odot}$ (Panagia 1973; Smith et al. 2002). Since the continuum emission is optically thick, this should be interpreted as a lower limit to the ionizing flux.

The source diameter of $15 \mathrm{AU}$ used in the model is dictated by the assumed electron temperature of $8000 \mathrm{~K}$, the measured flux densities, and the distance to Orion. A diameter of $15 \mathrm{AU}$ is, however, inconsistent with the observed size of SrcI, which is roughly 0 '.2, or $80 \mathrm{AU}$, along its major axis. This suggests that the emission region is clumpy or filamentary so that it does not fill the synthesized beam. But it is implausible that all of these clumps or filaments are perfectly sharp-edged, such that every one of them is optically thick even at $229 \mathrm{GHz}$. Generally, one expects a distribution of clumps to produce emission with a shallower spectral slope (Ignace \& Churchwell 2004).

One alternative is that the electron temperature is less than $8000 \mathrm{~K}$. The highest resolution measurements of SrcI that are available, $43 \mathrm{GHz}$ VLA observations with a 34 mas synthesized beam, imply that the source has a brightness temperature of only $1500 \mathrm{~K}$ (Reid et al. 2007). This is much cooler than is possible in an $\mathrm{H}$ II region; however, hydrogen is almost entirely neutral in dense gas at $T<4000 \mathrm{~K}$ (Reid \& Menten 1997).

\subsection{2. $H^{-}$Free-Free Emission from a Massive Disk?}

The absence of hydrogen recombination lines, $1500 \mathrm{~K}$ apparent brightness temperature, and a spectral index of $\sim 2$ are all difficult to explain if $\mathrm{SrcI}$ is a hypercompact $\mathrm{H}$ II region. The data more naturally fit an alternative hypothesis, first considered by Reid et al. (2007), that the emission from SrcI originates in a $T<4500 \mathrm{~K}$ disk, similar to the radio photospheres of Mira variables (Reid \& Menten 1997). The infrared spectrum of SrcI, seen via light reflected off of the neighboring nebulosity, suggests such an interpretation as well-Testi et al. (2010) find that the spectrum is characteristic of a low-gravity photosphere with effective temperature $3500-4500 \mathrm{~K}$. The close association of $\mathrm{SrcI}$ with $\mathrm{SiO}$ masers, also characteristic of Mira variables, further strengthens the case for this hypothesis.

As discussed by Reid \& Menten (1997), free-free emission at temperatures of about $1500 \mathrm{~K}$ in such a photosphere arises via the $\mathrm{H}^{-}$opacity, the scattering of electrons by neutral hydrogen atoms or molecules. The electrons are produced by collisional ionization of $\mathrm{Na}, \mathrm{K}$, and other metals. The $\mathrm{H}^{-}$opacity is roughly $10^{3}$ times smaller than the $p^{+} / e^{-}$opacity, so high densities $\left(>10^{11} \mathrm{~cm}^{-3}\right)$ typically are required to obtain optically thick emission.

Reid et al. (2007) were able to fit the $43 \mathrm{GHz}$ VLA observations of SrcI with models of $\mathrm{H}^{-}$emission from an $80 \mathrm{AU}$ diameter, $3 M_{\odot}$ disk. However, they found that such a large disk cannot be maintained at $\mathrm{T} \geqslant 1500 \mathrm{~K}$ if it is heated solely by 


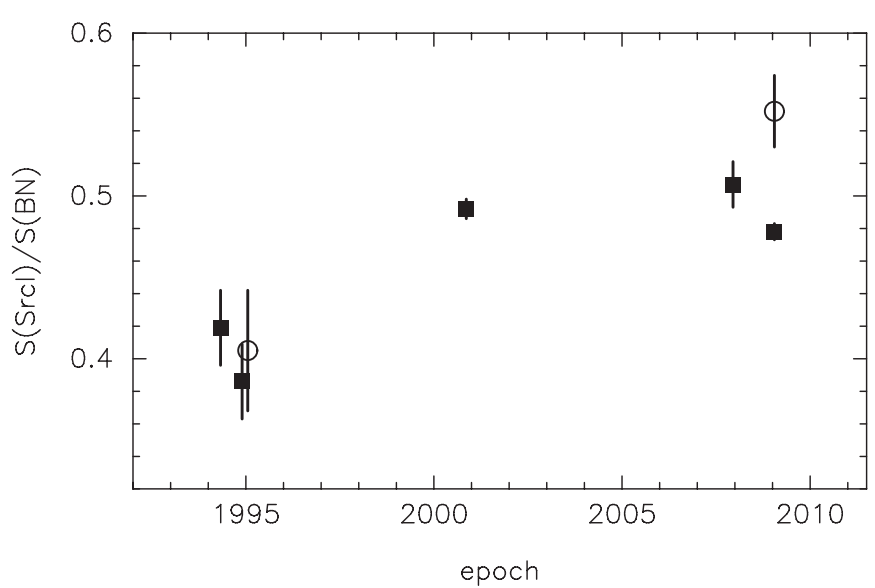

Figure 5. SrcI/BN flux density ratio vs. year, at $43 \mathrm{GHz}$ (filled squares) and at $86 \mathrm{GHz}$ (circles), derived from the data in Table 2 . This ratio should be immune to errors in the absolute flux scale, since the SrcI and BN are just $10^{\prime \prime}$ apart on the sky and are observed simultaneously. These data provide possible evidence that SrcI has brightened relative to BN since 1995.

a central star-a stellar luminosity $>10^{5} L_{\odot}$, greater than the luminosity of the entire Orion-KL region, would be required. They proposed that accretion processes might heat the disk locally. Testi et al. (2010) also find that the infrared spectrum of SrcI can be explained by emission from a disk around a $\sim 10 M_{\odot}$ protostar that is accreting at a few $\times 10^{-3} M_{\odot} \mathrm{yr}^{-1}$. However, their models show that the disk temperature falls below $1500 \mathrm{~K}$ for $\mathrm{r}>10 \mathrm{AU}$, and so cannot easily explain the $\mathrm{H}^{-}$free-free emission at larger radii.

In summary, the $\mathrm{H}^{-}$hypothesis explains the observational results in a natural way. The principal barrier to its acceptance is uncertainty over the mechanism by which such a large disk can be maintained at sufficiently high temperature. Whether the disk could be heated by an anomalously high accretion rate, a merging binary, magnetic effects, or some other mechanism remains a key question.

\subsubsection{Is SrcI Brightening?}

If accretion is responsible for much of SrcI's luminosity, then one might expect to see changes in its flux density on timescales of years. Although Goddi et al. (2011) found no evidence of flux variations at $43 \mathrm{GHz}$ over four epochs from 2000 to 2009, we are struck by the discrepancy in the $86 \mathrm{GHz}$ fluxes measured at BIMA in 1995 (34 \pm 5 mJy; Plambeck et al. 1995) and at CARMA in 2009 (50 \pm 5 mJy; Friedel \& Widicus Weaver 2011).

Generally, uncertainties in absolute flux densities are dominated by systematic effects - e.g., variations in telescope gains as a function of elevation, or uncertainties in the brightness temperature models of planets used as primary flux standards. The ratio of the flux densities of SrcI and BN should be immune to such effects because the two sources are just 10" apart and can be observed simultaneously. In Figure 5, we plot this ratio as a function of time for the 43 and $86 \mathrm{GHz}$ data in Table 2. Note that the error bars in this figure were calculated from the thermal noise in the original maps, not from the absolute flux uncertainties listed in Table 2. From these seven independent data sets, it appears that the ratio increased from about 0.4 in 1995 to 0.5 in 2009.

This result should be interpreted cautiously. Although the flux density ratio is unaffected by the absolute calibration scale, it is sensitive to the sampling of visibility data in the $(u, v)$ plane. It is also unclear whether SrcI or BN, or both, are varying; periodic variations in the infrared luminosity of $\mathrm{BN}$ were reported by Hillenbrand et al. (2001). Future careful monitoring of the flux densities of both SrcI and BN with the VLA and ALMA will be required to find conclusive evidence for source brightness variations.

\subsubsection{Vibrationally Excited $\mathrm{H}_{2} \mathrm{O}$}

The bright emission line in the spectrum of SrcI in Figure 1 is the $\mathrm{H}_{2} \mathrm{O} \mathrm{v}_{2}=1,5(5,0)-6(4,3)$ transition of $\mathrm{H}_{2} \mathrm{O}$ at $232.687 \mathrm{GHz}$, $3450 \mathrm{~K}$ above the ground state. This line was also recognized by Hirota et al. (2012) in the Orion Band 6 ALMA Science Verification data. With $0.6 \mathrm{~km} \mathrm{~s}^{-1}$ velocity resolution, the ALMA data show that the $\mathrm{H}_{2} \mathrm{O}$ line is double-peaked, like the $22 \mathrm{GHz} \mathrm{H}_{2} \mathrm{O}$ masers or the 43 and $86 \mathrm{GHz} \mathrm{SiO}$ masers toward SrcI.

In the $1^{\prime \prime} .5$ resolution ALMA maps, the $\mathrm{H}_{2} \mathrm{O}$ line is blended with a $\mathrm{HCOOCH}_{3}$ transition at $232.684 \mathrm{GHz}$, whereas in the 0.25 resolution CARMA map, the $\mathrm{HCOOCH}_{3}$ emission is resolved out. The $\mathrm{H}_{2} \mathrm{O}$ emission is unresolved by the CARMA beam, so it is clear that the line originates from the compact $\mathrm{SiO}$ maser zone close to SrcI, rather than the extended $2^{\prime \prime} \times 0$ 0.5 strip where $22 \mathrm{GHz} \mathrm{H}_{2} \mathrm{O}$ masers are found (Gaume et al. 1998). The $\mathrm{SiO} v=2$ masers also originate in energy levels $3500 \mathrm{~K}$ above the ground state; Goddi et al. (2009) find that these masers can be excited in gas with kinetic temperature $>2000 \mathrm{~K}$.

The same transition of vibrationally excited $\mathrm{H}_{2} \mathrm{O}$ was detected toward the evolved stars VY CMa and W Hya by Menten \& Melnick (1989). Like SrcI, both of these stars have $\mathrm{SiO}$ and $22 \mathrm{GHz} \mathrm{H}_{2} \mathrm{O}$ masers.

\section{SUMMARY}

CARMA $229 \mathrm{GHz}$ maps of Orion-KL with up to 0.' 14 angular resolution clearly distinguish the ionized envelopes of SrcI and $\mathrm{BN}$ from the surrounding molecular cloud. The principal results are as follows:

1. The integrated flux densities of SrcI and BN at $229 \mathrm{GHz}$ are 310 and $240 \mathrm{mJy}$, respectively, with a probable uncertainty of $\pm 15 \%$. The BN flux density we measure with 0 '. 14 resolution is a factor of two greater than that derived by Galván-Madrid et al. (2012) from the ALMA Science Verification data with a $1 . .3 \times 0$ ". 6 synthesized beam. We suspect that the flux density of $\mathrm{BN}$ is depressed in the ALMA map because of negative sidelobes from poorly sampled extended emission. These large scale structures are filtered out more effectively in the higher resolution CARMA data.

2. SrcI's flux density is proportional to $v^{2}$ from 43 to $229 \mathrm{GHz}$ - it appears to be an optically thick black body. By comparison, BN's flux density scales as $v^{1.3}$ up to about $100 \mathrm{GHz}$, then flattens; its free-free continuum is becoming optically thin at $229 \mathrm{GHz}$.

3. SrcI is elongated at P.A. 140 degrees in the CARMA images. Its deconvolved size is similar at 229 and $43 \mathrm{GHz}$ - further evidence that it is optically thick.

4. The $\mathrm{H} 30 \alpha$ recombination line is not detected toward SrcI, though it is bright toward BN. The absence of this recombination line is a third piece of evidence that SrcI is optically thick, or that it is not an $\mathrm{H}$ II region at all.

5. The $\mathrm{v}_{2}=1,5(5,0)-6(4,3)$ transition of $\mathrm{H}_{2} \mathrm{O}$ at $232.687 \mathrm{GHz}$, $3450 \mathrm{~K}$ above the ground state, is prominent toward SrcI. The line emission originates close to the central star, not in the larger zone where $22 \mathrm{GHz} \mathrm{H}_{2} \mathrm{O}$ masers are found. 
6. While our observational results do not rule out the possibility that $\mathrm{SrcI}$ is an exceptionally dense hypercompact $\mathrm{H}$ II region, they are more easily explained by models of free-free emission via the $\mathrm{H}^{-}$opacity in a $T<4500 \mathrm{~K}$ disk, as modeled by Reid et al. (2007).

7. A model of a spherically symmetric hypercompact H II region with a diameter of about $15 \mathrm{AU}$ and electron density $n_{e} \sim 5 \times 10^{7} \mathrm{~cm}^{-3}$ matches both the continuum and the recombination line intensities toward $\mathrm{BN}$.

8. The excitation parameter $U \sim 5 \mathrm{pc} \mathrm{cm}^{-2}$ derived for $\mathrm{BN}$ implies that its Lyman continuum flux is $5 \times$ $10^{45}$ photons $\mathrm{s}^{-1}$, consistent with that expected from a zero age main sequence $B$ star with a total luminosity of $(0.5-1) \times 10^{4} L_{\odot}$.

9. The $\mathrm{H} 53 \alpha$ recombination line in $\mathrm{BN}$ is predicted to have a collisionally broadened linewidth of $200 \mathrm{~km} \mathrm{~s}^{-1}$. Presumably, the $\mathrm{H} 53 \alpha$ emission observed by Rodríguez et al. (2009), with a linewidth of about $40 \mathrm{~km} \mathrm{~s}^{-1}$, originates primarily from lower density ionized gas farther from the central star.

10. The $V_{\mathrm{LSR}}$ of $\mathrm{BN}$ is $23.2 \pm 0.5 \mathrm{~km} \mathrm{~s}^{-1}$, based on the central velocity of the $231.9 \mathrm{GHz} \mathrm{H} 30 \alpha$ line, which is little affected by pressure broadening or continuum opacity.

11. Published flux densities at both 43 and $86 \mathrm{GHz}$ provide tentative evidence that $\mathrm{SrcI}$ brightened with respect to $\mathrm{BN}$ between 1994 and 2009.

Future ALMA observations of Orion-KL at submillimeter wavelengths will provide a definitive test of the emission mechanism in SrcI. If SrcI is an H II region, then it must become optically thin at sufficiently high frequencies. If the source were still optically thick at $650 \mathrm{GHz}$, for example, then an O-star

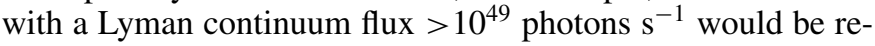
quired to maintain its ionization, but the luminosity of such a star, $>10^{5} L_{\odot}$ (Panagia 1973), would exceed the measured luminosity of the entire Orion-KL nebula. Then, at frequencies where the continuum is optically thin, hydrogen recombination lines must be detectable if hydrogen is ionized. If these recombination lines are detected, then the free-free hypothesis is confirmed. If not, or if the continuum flux density continues to increase following a $v^{2}$ law, the emission must be attributable to the $\mathrm{H}^{-}$mechanism.

Submillimeter observations of $\mathrm{BN}$, as well as more sensitive observations of its $\mathrm{cm}$ and $\mathrm{mm}$ wavelength recombination lines, will be useful in constraining models of the hypercompact $\mathrm{H}$ II region around this star, and in tracking small changes in the $\mathrm{SrcI} / \mathrm{BN}$ flux density ratio that could be an indication of time variable accretion onto these objects.

Support for CARMA construction was derived from the states of California, Illinois, and Maryland, the James S. McDonnell Foundation, the Gordon and Betty Moore Foundation, the Kenneth T. and Eileen L. Norris Foundation, the University of
Chicago, the Associates of the California Institute of Technology, and the National Science Foundation. Ongoing CARMA development and operations are supported by the National Science Foundation under a cooperative agreement, and by the CARMA partner universities.

Facility: CARMA

\section{REFERENCES}

Allen, D. A., \& Burton, M. G. 1993, Natur, 363, 54

Bally, J., Cunningham, N. J., Moeckel, N., et al. 2011, ApJ, 727, 113

Beuther, H., Zhang, Q., Greenhill, L. J., et al. 2004, ApJL, 616, L31

Beuther, H., Zhang, Q., Reid, M. J., et al. 2006, ApJ, 636, 323

Brocklehurst, M., \& Seaton, M. J. 1972, MNRAS, 157, 179

Chandler, C. J., \& Wood, D. O. S. 1997, MNRAS, 287, 445

De Buizer, J. M., Morris, M. R., Becklin, E. E., et al. 2012, ApJL, 749, L23

Doi, T., O’Dell, C. R., \& Hartigan, P. 2002, AJ, 124, 445

Eisner, J. A., Plambeck, R. L., Carpenter, J. M., et al. 2008, ApJ, 683, 304

Felli, M., Churchwell, E., Wilson, T. L., \& Taylor, G. B. 1993a, A\&AS, 98, 137

Felli, M., Taylor, G. B., Catarzi, M., Churchwell, E., \& Kurtz, S. 1993b, A\&AS, 101,127

Forbrich, J., Menten, K. M., \& Reid, M. J. 2008, A\&A, 477, 267

Friedel, D. N., \& Widicus Weaver, S. L. 2011, ApJ, 742, 64

Galván-Madrid, R., Goddi, C., \& Rodríguez, L. F. 2012, A\&A, 547, L3

Garay, G., Moran, J. M., \& Reid, M. J. 1987, ApJ, 314, 535

Gaume, R. A., Wilson, T. L., Vrba, F. J., Johnston, K. J., \& Schmid-Burgk, J. 1998, ApJ, 493, 940

Goddi, C., Greenhill, L. J., Chandler, C. J., et al. 2009, ApJ, 698, 1165

Goddi, C., Humphreys, E. M. L., Greenhill, L. J., Chandler, C. J., \& Matthews, L. D. 2011, ApJ, 728, 15

Gómez, L., Rodríguez, L. F., Loinard, L., et al. 2008, ApJ, 685, 333

Hillenbrand, L. A., Carpenter, J. M., \& Skrutskie, M. F. 2001, ApJL, 547, L53

Hirota, T., Kim, M. K., \& Honma, M. 2012, ApJL, 757, L1

Ignace, R., \& Churchwell, E. 2004, ApJ, 610, 351

Kim, M. K., Hirota, T., Honma, M., et al. 2008, PASJ, 60, 991

Matthews, L. D., Greenhill, L. J., Goddi, C., et al. 2010, ApJ, 708, 80

Menten, K. M., \& Melnick, G. J. 1989, ApJL, 341, L91

Menten, K. M., \& Reid, M. J. 1995, ApJL, 445, L157

Menten, K. M., Reid, M. J., Forbrich, J., \& Brunthaler, A. 2007, A\&A, 474, 515

Mezger, P. G., Smith, L. F., \& Churchwell, E. 1974, A\&A, 32, 269

Morino, J.-I., Yamashita, T., Hasegawa, T., \& Nakano, T. 1998, Natur, 393, 340

Panagia, N. 1973, AJ, 78, 929

Pérez, L. M., Lamb, J. W., Woody, D. P., et al. 2010, ApJ, 724, 493

Plambeck, R. L., Wright, M. C. H., Friedel, D. N., et al. 2009, ApJL, 704, L25

Plambeck, R. L., Wright, M. C. H., Mundy, L. G., \& Looney, L. W. 1995, ApJL, 455, L189

Reid, M. J., \& Menten, K. M. 1997, ApJ, 476, 327

Reid, M. J., Menten, K. M., Greenhill, L. J., \& Chandler, C. J. 2007, ApJ, 664,950

Rodríguez, L. F., Poveda, A., Lizano, S., \& Allen, C. 2005, ApJL, 627, L65

Rodríguez, L. F., Zapata, L. A., \& Ho, P. T. P. 2009, ApJ, 692, 162

Rohlfs, K., \& Wilson, T. L. 2000, Tools of Radio Astronomy (3rd ed.; New York: Springer)

Schaller, G., Schaerer, D., Meynet, G., \& Maeder, A. 1992, A\&AS, 96, 269

Scoville, N., Kleinmann, S. G., Hall, D. N. B., \& Ridgway, S. T. 1983, ApJ, 275, 201

Smith, L. J., Norris, R. P. F., \& Crowther, P. A. 2002, MNRAS, 337, 1309

Testi, L., Tan, J. C., \& Palla, F. 2010, A\&A, 522, A44

Vacca, W. D., Garmany, C. D., \& Shull, J. M. 1996, ApJ, 460, 914

Wright, A. E., \& Barlow, M. J. 1975, MNRAS, 170, 41

Zapata, L. A., Loinard, L., Schmid-Burgk, J., et al. 2011, ApJL, 726, L12 